\title{
Numerical simulation on wind pressure transients in high speed train tunnels
}

\author{
S.-W. Nam \\ Department of High Speed Train, \\ Korea Railroad Research Institute, Korea
}

\begin{abstract}
When a train passes through a tunnel with high speed, pressure waves are generated due to the entry of the front and the rear of the train respectively. These pressure waves are alternately propagated and reflected at the exit and entrance of the tunnel. Severe pressure transients cause the repeated fatigue load to the car-body and tunnel wall, ear-discomfort to passengers, and micro pressure wave to the surroundings of the tunnel exit. To clarify quantitative pressure change in the tunnel is very important for the optimal design of the tunnel size. Partial differential equations also can be converted into ordinary differential equations by using the method of characteristics. In this study, we have developed a simulation program to calculate pressure transients in a railroad tunnel. The method of characteristics based on a fixed mesh system is used to solve the governing equations. By using this program, we have predicted pressure transients in KTX (Korea Train eXpress)-tunnel and compared with experimental results that are obtained in Unju tunnel with KTX high speed trains. The results of calculation coincide with the ones of the Unju tunnel tests, and the pressure transient mechanism was made clear. To analyze the effects of train speed and blockage ratio, we have conducted parametric simulations. The results show that pressure rise in a tunnel is proportional to the square of train speed and to the blockage of train/tunnel cross sectional area.
\end{abstract}

Keywords: wind pressure, high speed train, tunnel flow, pressure wave.

\section{Introduction}

When a train enters and passes through a tunnel with high speed, a compression wave is generated due to the entry of the train head and an expansion wave is 
generated due to the entry of the train tail. These pressure waves propagate along the tunnel and reflect in turn at the exit and entrance of the tunnel. So, a complex wave phenomenon appears in the railway tunnel. Severe pressure transients cause the repeated fatigue load on the car body and tunnel wall, ear-discomfort to passengers, and micro pressure wave to the environment [1]. Flow in the long tunnel can be assumed as a one-dimensional internal pipe flow under the assumption that variables in a constant cross section of the tunnel are the same. Because pressure increase in the tunnel is proportional to the square of train speed, it is very important to design the optimal tunnel design, drag reduction of train, worker's safety in tunnel, and stress increase of car body. In order to understand complex pressure transient phenomena in a tunnel, real train test is the most effective method. But, to do real train experiment is a time and manpower consuming approach.

So, many researchers choose the numerical simulation to replace that. $\mathrm{Wu}[1]$ and Suzuki [2] used 3-dimensional model to clarify pressure transient in a tunnel and Kwon et al. [3] used an axisymmetric one. But, these methods need lots of computational mesh and long computing time in the case of a long tunnel model.

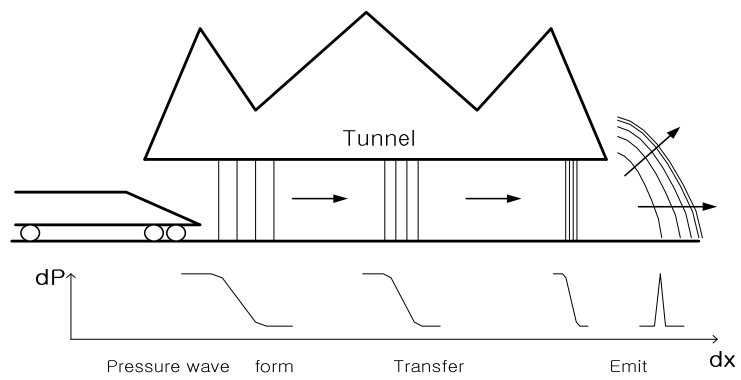

Figure 1: Pressure transient in a tunnel.

Yamamoto [4] set a one-dimensional model under the assumption that flows in the same cross section of a tunnel are almost constant. After that, Maeda improved the methods and Vardy and Dayman [5] and Kim [6] applied to the analysis of pressure transient in a tunnel. Almost all the precedent researches used the variable mesh system to calculate the characteristics curves and the position of train. In this study, fixed mesh system is adopted. Simulation results with characteristics method are compared with that of real train experiment and showed good coincidence quantitatively and qualitatively.

\section{Numerical calculation and details}

One-dimensional governing equations can be written as follows under the assumption that variables in constant cross section of a tunnel are the same.

$$
\frac{\partial u}{\partial t}+u \frac{\partial u}{\partial x}+\frac{1}{\rho} \frac{\partial p}{\partial x}=f
$$




$$
a^{2} \frac{\partial u}{\partial x}+\frac{1}{\rho}\left(\frac{\partial p}{\partial t}+u \frac{\partial p}{\partial x}\right)=(\gamma-1) \varphi
$$

These partial differential equations also converted to ordinary differential ones following to characteristics curves of $\mathrm{C}+$ and $\mathrm{C}$ - [7].

$$
\begin{aligned}
& \frac{d}{d t}(p+\rho a u)=(\gamma-1) \rho \varphi+\rho a f \\
& \frac{d}{d t}(p-\rho a u)=(\gamma-1) \rho \varphi-\rho a f
\end{aligned}
$$

Friction force and energy dissipation can be described as following the position of the train (shown in Figs 2 and 3).

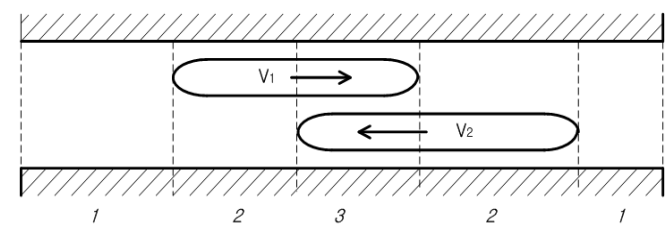

Figure 2: Calculation domain.

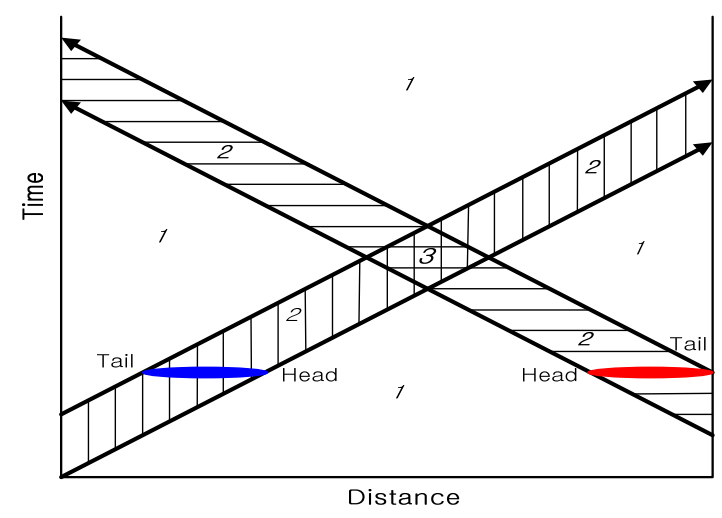

Figure 3: Distance-time diagram.

Domain 1:

$$
\begin{gathered}
f_{1}=-\frac{\lambda}{2 d} u_{1}\left|u_{1}\right| \\
\varphi_{1}=\frac{\lambda}{2 d}\left|u_{1}\right|^{3}
\end{gathered}
$$


Domain 2:

$$
\begin{array}{r}
f_{2}=-\frac{\lambda}{2 d} \frac{1}{1-R} u_{1}\left|u_{1}\right|-\frac{\lambda^{\prime}}{2 d^{\prime}} \frac{R}{1-R}\left(u_{2}-V_{1}\right)\left|u_{2}-V_{1}\right| \\
\varphi_{2}=\frac{\lambda}{2 d} \frac{1}{1-R}\left|u_{2}\right|^{3}+\frac{\lambda^{\prime}}{2 d^{\prime}} \frac{R}{1-R}\left|u_{2}-V_{1}\right|^{3}
\end{array}
$$

Domain 3:

$$
\begin{gathered}
f_{3}=-\frac{\lambda}{2 d} \frac{1}{1-2 R} u_{3}\left|u_{3}\right|-\frac{\lambda^{\prime}}{2 d^{\prime}} \frac{R}{1-2 R}\left(u_{3}-v_{1}\right)\left|u_{3}-v_{1}\right|-\frac{\lambda^{\prime}}{2 d^{\prime}} \frac{R}{1-2 R}\left(u_{3}-v_{2}\right)\left|u_{3}-v_{2}\right| \\
\varphi_{3}=\frac{\lambda}{2 d} \frac{1}{1-2 R}\left|u_{3}\right|^{3}+\frac{\lambda^{\prime}}{2 d^{\prime}} \frac{R}{1-2 R}\left|u_{3}-v_{1}\right|^{3}+\frac{\lambda^{\prime}}{2 d^{\prime}} \frac{R}{1-2 R}\left|u_{3}-v_{2}\right|^{3}
\end{gathered}
$$

According to the railroad track and train type, a proper friction coefficient can be adopted and boundary conditions at a tunnel inlet and outlet are as follows:

Tunnel inlet:

$$
\begin{gathered}
p=-\frac{1}{2} \rho u^{2}: \text { in case of } u>0 \\
p=0 \quad: \text { in case of } u \leq 0
\end{gathered}
$$

Tunnel outlet:

$$
\begin{aligned}
& p=-\frac{1}{2} \rho u^{2}: \text { in case of } u<0 \\
& p=0 \quad: \text { in case of } u \geq 0
\end{aligned}
$$

In this study, fixed mesh system is used to calculate velocity and pressure whereas preceding studies used variable mesh one. In the fixed mesh system, we don't need to calculate intersection point of two characteristics curves every time.

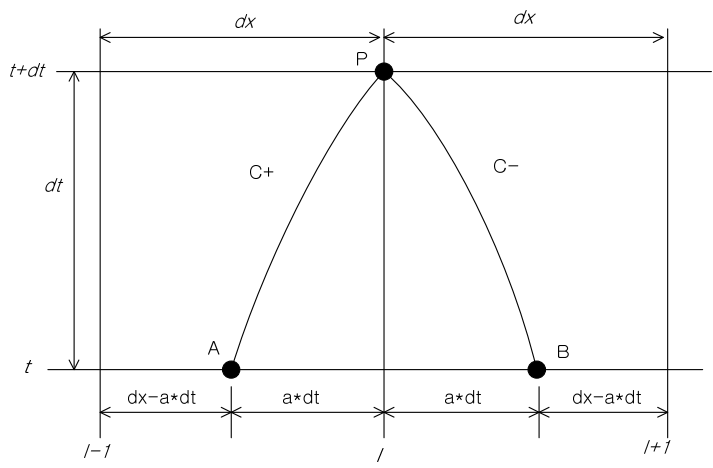

Figure 4: Fixed rectangular mesh.

Between point $\mathrm{P}$ and $\mathrm{A}$ on the curve $\mathrm{C}+$, point $\mathrm{P}$ and $\mathrm{B}$ on the curve $\mathrm{C}$-, Riemann invariant can be obtained. Pressure and velocity of point $\mathrm{P}$ on time step $t+\Delta t$ also are calculated by using values of grid point $i, i+1$, and $i-1$ on time step $t$. 
Dependent variables at point $\mathrm{A}$ and $\mathrm{B}$ are calculated by interpolating with values of mesh i, i-1, and $\mathrm{i}+1$. Then, Pressure and velocity of point $\mathrm{P}$ on the characteristics curves of $\mathrm{C}+$ and $\mathrm{C}$ - are obtained as follows [8];

$$
\begin{aligned}
U_{P} & =\frac{Q_{A}-Q_{B}}{2 \rho a} \\
P_{P} & =\frac{Q_{A}+Q_{B}}{2}
\end{aligned}
$$

where

$$
\begin{aligned}
& Q_{A}=[(\gamma-1) \rho \varphi+\rho a f]_{A} d t+P_{A}+\rho a U_{A} \\
& Q_{B}=[(\gamma-1) \rho \varphi-\rho a f]_{B} d t+P_{B}-\rho a U_{B}
\end{aligned}
$$

Boundary conditions at the train head and tail are also written by simultaneous equations.

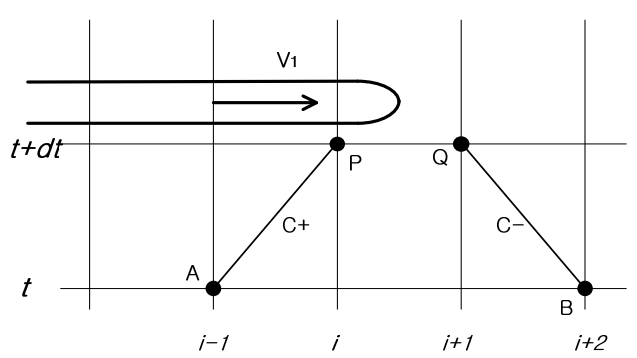

Figure 5: Boundary condition of train head.

In Fig. 5, when the train head is located in the position between $\mathrm{P}$ and $\mathrm{Q}$, simultaneous equations can be applied.

$$
\begin{gathered}
(P+\rho a U)_{P}-(P+\rho a U)_{A}=0 \\
(P-\rho a U)_{Q}-(P-\rho a U)_{B}=0 \\
(1-R)\left(U_{P}-V_{1}\right)=U_{Q}-V_{1} \\
P_{P}+\frac{1}{2} \rho\left(U_{P}-V_{1}\right)^{2}=P_{Q}+\frac{1}{2} \rho\left(U_{Q}-V_{1}\right)^{2}
\end{gathered}
$$

So, pressure and velocity for each point, P and Q, are obtained simultaneously. And, calculation time step $\Delta t$ is determined by grid size and sound speed as follows.

$$
\Delta t \leq \frac{\Delta x}{C a}
$$


According to the Courant-Friedrichs-Lewy condition, constant $\mathrm{C}$ set as 1-2. Overall procedure of calculation is as in the following. At first, divide the tunnel into equally-spaced meshes and assign initial conditions of pressure and velocity as 0 at time step $t=0$. Calculate pressure and velocity of internal meshes of the tunnel by using Eqs. (13) and (14) at time step $t+d t$. At the same time, calculate the position of train head and tail. And then, apply boundary conditions of Eqs. (15)-(18) to connection points of train head and tail. Renew inlet and outlet condition of the tunnel of Eqs. (11) and (12). Repeat these procedures until the train goes through the tunnel exit.

\section{Results}

In order to compare simulation results with experimental ones, the real train experiment using Korean high speed train, KTX, has been conducted in Kyungbu line. Comparison target is Unju tunnel that measures $4030 \mathrm{~m}$ in length and KTX train enters the tunnel with travelling speeds of $265 \mathrm{~km} / \mathrm{h}$. Side pressure variation on the head of train going through Unju tunnel is shown in Fig. 7. There is atmospheric pressure difference of $380 \mathrm{~Pa}$ between the tunnel inlet and outlet because the tunnel slopes gently down by $8 \%$. When the train enters and passes through the tunnel with high speed, a compression wave is generated due to the entry of the train head and an expansion wave is generated due to the entry of the train tail. These pressure waves propagate along the tunnel and reflect in turn at the exit and entrance of the tunnel. So, complex phenomenon of pressure wave appears in the railway tunnel. When the train meets with an expansion wave as point 1, a pressure decrease occurs whereas pressure increases with compression wave as point 2. Comparison between simulation and experiment of pressure transients is shown in Fig. 7.

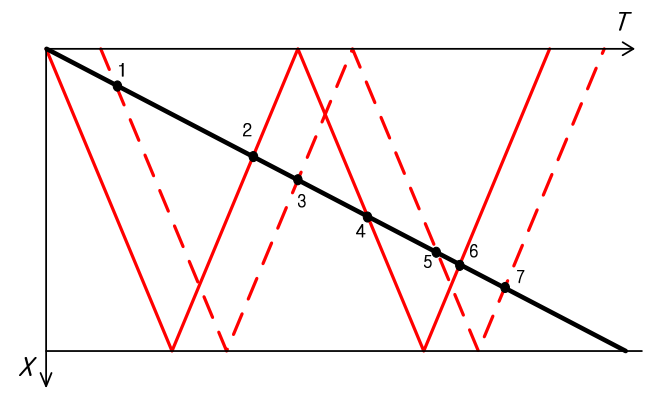

Figure 6: X-t diagram. 


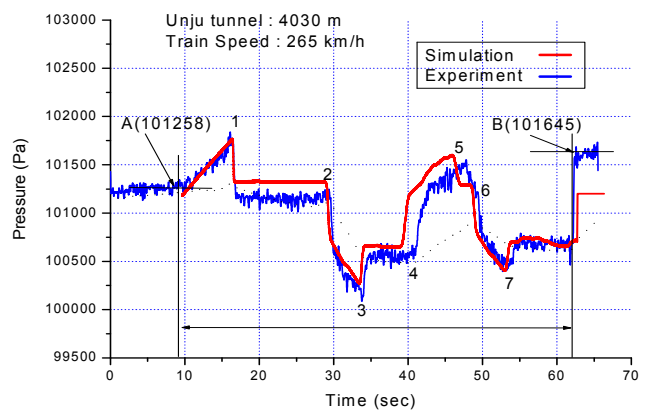

Figure 7: Comparison between simulation and experiment.

In the figure, the simulation result is represented by a red line and experimental one is a blue line. The results of simulation correspond with that of experiment qualitatively and quantitatively. In the latter half of tunnel, there is some discrepancy in pressure changing pattern. It is considered that pressure waves propagate with the speed of sound in simulation but these waves are disturbed by various obstacles in real tunnel, for example, surface roughness of tunnel, by-pass tunnel for fire and ventilation, maintenance facilities, sleepers, ballast, and electric power catenary equipment etc.

After the train enters the tunnel, pressure increases until train head meets with expansion wave at point 1 . The range of pressure increase of simulation and experiment are $650 \mathrm{~Pa}$ and $700 \mathrm{~Pa}$ respectively. And the range between point 2 and point 3 are $1162 \mathrm{~Pa}$ and $1306 \mathrm{~Pa}$.

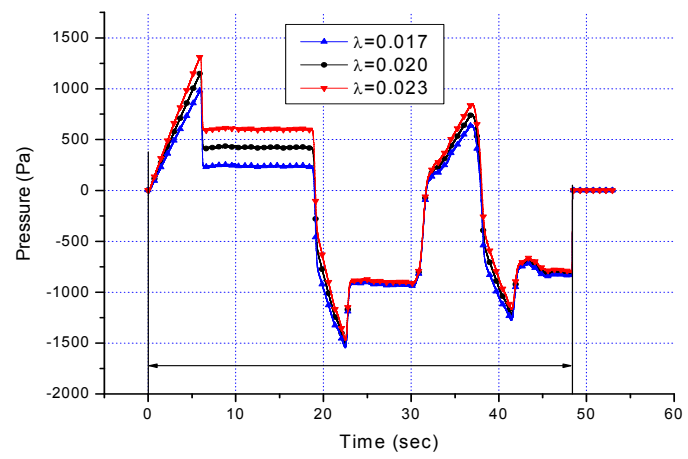

Figure 8: Effects of friction coefficient.

Figure 8 shows the effects of friction coefficient of the train surface. It is considered that small value of friction coefficient means a streamline type of train shape. From the figure, we know that small friction coefficient for train 
surface results in the small pressure increase when train enter tunnel. So design for streamlined shape is an effective method to reduce pressure increase in a tunnel.

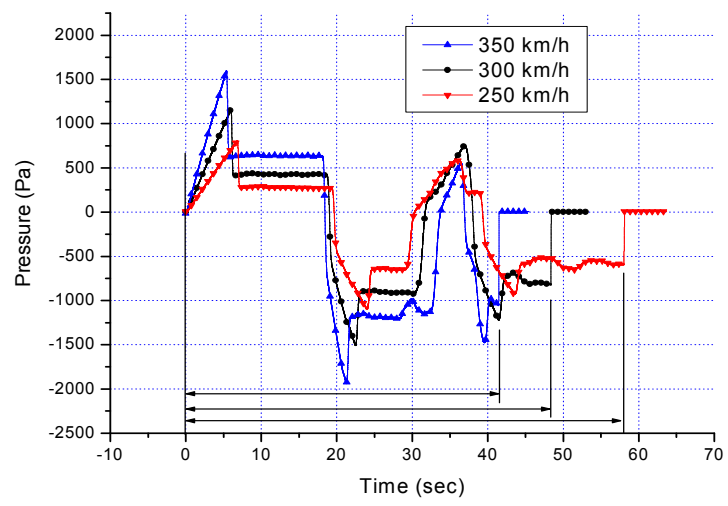

Figure 9: Effects of train velocity.

Figure 9 represents the effects of velocity of train entering tunnel. In case of train speed of $250 \mathrm{~km} / \mathrm{h}, 300 \mathrm{~km} / \mathrm{h}$, and $350 \mathrm{~km} / \mathrm{h}$, pressure increase are $772 \mathrm{~Pa}$, $1130 \mathrm{~Pa}$, and $1525 \mathrm{~Pa}$ respectively. So pressure increase at tunnel inlet part is approximately proportional to the square of train velocity.

Figure 10 compared the effects of tunnel cross sectional area for pressure variation. Three cases for tunnel area of $107 \mathrm{~m}^{2}, 100 \mathrm{~m}^{2}$, and $90 \mathrm{~m}^{2}$ have been chosen to compare the effects to pressure variation.

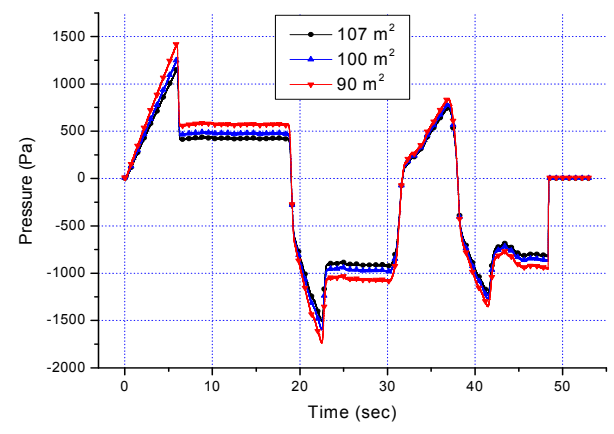

Figure 10: Effects of tunnel cross section area.

It is clear that the range of pressure increases in case of $107 \mathrm{~m}^{2}$ is smaller than that of small tunnels. To make a tunnel big it is effective to reduce pressure increase in the tunnel, but the cost to construct the tunnel reversely increases. 


\section{Conclusions}

In this study, we have developed a simulation program to calculate internal pressure transients in railroad tunnel. Characteristics method based on fixed mesh system is used to solve one-dimensional governing equations. By using this program, we have predicted pressure transients in KTX (Korea Train eXpress)-tunnel. Comparisons are also made with high speed train, KTX, measurements obtained in Unju tunnel. The results of calculation coincide in ones of the Unju tunnel tests, and pressure transient mechanism was made clear. It is considered that small value of friction coefficient means streamline type of train shape. From the results, small friction coefficient for train surface results in the small pressure increase when train enter tunnel. So design for streamlined shape is effective method to reduce pressure increase in tunnel.

To analyze the effects of train speed and blockage ratio, we have conducted parametric simulations. Pressure rise in tunnel is proportional to the square of train speed and to the blockage of train/tunnel cross sectional area.

In the following study, it is necessary to consider variable train speed in tunnel and the effects of by-pass tunnel for practical model.

\section{Acknowledgement}

This study was supported by research funds from Korea Rail Network Authority.

\section{References}

[1] P.K.H. Wu. Prediction of Pressure wave generation by high-speed train entering tunnel using a commercial CFD code, BHR Group 2000 Vehicle Tunnels, 1, pp. 767-777, 2000.

[2] M. Suzuki. Aerodynamic Force acting on Train in Tunnel, RTRI Report, 14(9), pp. 37-42, 2000.

[3] H.B. Kwon, T.Y. Kim, D.H. Lee, M.S. Kim. Numerical simulation of unsteady compressible flows induced by a high-speed train passing through a tunnel, Proc. Inst. Mech. Engrs. Part F: J Rail and Rapid Transit., 217, pp. 111-124, 2003.

[4] A. Yamamoto. Aerodynamics of Train and Tunnel, RTRI Report, 1230, pp. 1-70, 1983.

[5] A.E. Vardy, B. Dayman. Alleviation of Tunnel Entry Pressure Transients: Theoretical Modelling and Experimental Correlation, 3rd Int. Symp. on the Aerodynamics and Ventilation of Vehicle Tunnels, pp. 363-375, 1979.

[6] Heuy-Dong Kim. Aerodynamic Analysis of a Train Running in a Tunnel(1)Aerodynamics of One-Train, J. Korean Society of Mechanical Engineers (B), 21(8), pp. 963-972, 1997.

[7] G. Rudinger. Wave Diagrams for Nonsteady Flow in Ducts, D. Van Nostrand Co. Inc., New York. pp. 8-19, 1955.

[8] C.Y. Chow. An Introduction to Computational Fluid Mechanics, John Wiley \& Sons, Inc. pp. 180-195, 1979. 